\title{
IgG4-Related Sclerosing Cholangitis and Primary Sclerosing Cholangitis
}

\author{
Atsushi Tanaka \\ Department of Medicine, Teikyo University School of Medicine, Tokyo, Japan
}

Sclerosing cholangitis (SC) is defined as a condition with progressive stenosis and destruction of the bile ducts due to diffuse inflammation and fibrosis and currently includes three categories: primary sclerosing cholangitis (PSC), secondary cholangitis, and IgG4-related sclerosing cholangitis (IgG4-SC). SC categories share similar clinical features, such as cholestasis. Patients with SC present with cholestatic symptoms, including jaundice and pruritus, and blood tests reveal elevation of cholestatic enzymes. Cholangiography, endoscopic or magnetic, is inevitably required for making a diagnosis. Although the presentation of IgG4-SC and PSC are similar, the comorbidities, treatment response, and outcomes differ significantly, and therefore, it is strongly advisable to be familiar with these two diseases to make a correct diagnosis. Differentiation of cholangiocarcinoma from lgG4-SC and PSC is also extremely important. In this review, the clinical characteristics, comorbidities, treatment and outcomes of IgG4-SC and PSC will be outlined based on experience mainly from Japan. (Gut Liver 2019;13:300-307)

Key Words: IgG4; Cholangiography; Cholangitis; Corticosteroid

\section{INTRODUCTION}

Sclerosing cholangitis (SC) is defined as a condition with progressive stenosis and destruction of the bile ducts due to diffuse inflammation and fibrosis. ${ }^{1,2} \mathrm{SC}$ was formally classified into two categories: primary sclerosing cholangitis (PSC) with unknown etiology, and secondary SC caused by a variety of pathological conditions, including postoperative biliary stenosis, bacterial cholangitis, and choledocholithiasis. ${ }^{3}$ Recently another etiology of SC, IgG4-related sclerosing cholangitis (IgG4-SC), was established as a clinical phenotype in the biliary tree of a systemic IgG4-related disease (IgG4-RD). Thus, SC is now categorized as
PSC, IgG4-SC, and secondary SC.

In 2015, we performed a nationwide, multicenter survey by sending questionnaires to physicians in order to elucidate the present status of PSC and IgG4-SC in Japan. Comprehensive information on 435 and 527 patients with PSC and IgG4-SC, respectively, were recorded, and we have already published these results. ${ }^{4,5}$ This review, with citing data from these works, will outline the epidemiology and demographics, clinical characteristics, management, and outcomes of PSC and IgG4-SC, mainly based on recent literature and our experience in Japan.

\section{IgG4-SCLEROSING CHOLANGITIS}

IgG4-SC is also known as IgG4-associated cholangitis (IAC) mainly in the European literature. IgG4-SC is a biliary tract manifestation of IgG4-RD, characterized by systemic, inflammatory, sclerosing lesions with massive infiltrations of IgG4positive lymphocytes involving many organs including the eye; salivary, and lacrimal glands; lungs; pancreas; retroperitoneum, kidneys; and vascular systems. ${ }^{7-11}$ In particular, IgG4-SC is frequently found associated with pancreatic involvement in IgG4$\mathrm{RD}$, a condition termed autoimmune pancreatitis (AIP). ${ }^{12}$ AIP and IgG4-SC are relatively new clinical entities, established by the landmark studies of Hamano et al. ${ }^{13}$ in 2001 and Kamisawa et al. ${ }^{14}$ in 2003. The clinical diagnostic criteria of IgG4-SC were established by the Japanese Biliary Association in 2012 (Table $1)^{15}$ to facilitate its correct diagnosis and differentiation from PSC or cholangiocarcinoma. Conversely, the HISORt criteria, consisting of histology, imaging, serology, other organ involvement (OOI) and response to therapy, have frequently been used for the diagnosis of IgG4-SC (or IAC) in the United States and Europe. ${ }^{16-18}$ The HISORt also consist of histology, imaging, serology, OOI of IgG4-RD, and response to steroid therapy ${ }^{19}$ but were originally designed for the diagnosis of AIP.

Correspondence to: Atsushi Tanaka (https://orcid.org/0000-0002-6358-5283)

Department of Medicine, Teikyo University School of Medicine, 2-11-1, Kaga, Itabashi-ku, Tokyo 173-8605, Japan

Tel: +81-3-3964-1211, Fax: +81-3-3964-6627, E-mail: a-tanaka@med.teikyo-u.ac.jp

Received on February 7, 2018. Revised on July 2, 2018. Accepted on July 2, 2018. Published online September 13, 2018 pISSN 1976-2283 eISSN 2005-1212 https://doi.org/10.5009/gnl18085

@ This is an Open Access article distributed under the terms of the Creative Commons Attribution Non-Commercial License (http://creativecommons.org/licenses/by-nc/4.0) which permits unrestricted non-commercial use, distribution, and reproduction in any medium, provided the original work is properly cited. 


\section{Epidemiology and demographics}

Despite epidemiological data of IgG4-SC in Japan are lacking, these can be extrapolated from those obtained for AIP. In 2011, Kanno et al. ${ }^{20}$ carried out a clinico-epidemiological survey of AIP in Japan, yielding an overall prevalence of 4.6 per 100,000 population, and an annual incidence of 1.4 per 100,000 population. In this study, the prevalence of IgG4-SC in patients with AIP was reported to be $39 \%$, and thus an overall prevalence and an annual incidence of patients who have both AIP and IgG4SC are estimated as 1.8 and 0.5 per 100,000 population, respectively. Furthermore, our nationwide survey of IgG4-SC in 2015 demonstrated that the proportion of patients diagnosed as having both IgG4-SC and AIP was 87\% of all IgG4-SC cases. ${ }^{5}$ Collectively, the overall incidence and annual prevalence of IgG4SC in Japan were calculated to be 2.1 and 0.63 per 100,000 population, respectively, which are less than half of those of AIP.

The demographics of patients with IgG4-SC are summarized in Table 2. IgG4-RD is generally a male-dominant disease, and indeed male patients were also dominant in other cases series of IgG4-SC from the United States ${ }^{18}$ and United Kingdom. ${ }^{21}$ The age at presentation was also similar among the three reports, indicating that patients in their 60s were at the highest risk of developing IgG4-SC. In Fig. 1A, the age distributions at presentation are shown for 527 cases of IgG4-SC in Japan. Ages ranged from 23.0 to 88.5 years and unlike PSC, which can be difficult to differentiate from IgG4-SC in terms of biliary imaging, no patient developed IgG4-SC in childhood or adolescence. The median age at diagnosis was 66.2 years.

Although case-controls studies to clarify the etiology of this enigmatic disease have not been carried out, preliminary data have suggested a role for environmental triggers in the development of IgG4-SC and AIP. ${ }^{22}$ A questionnaire-based study revealed that a history of "blue-collar" work was noted in 88\% of patients with IgG4-SC and/or AIP in the Amsterdam cohort, and in $61 \%$ of patients with IgG4-SC and/or AIP in the Oxford cohort, both of which were much higher than the rate observed in patients with PSC. ${ }^{22}$ Occupational antigens such as solvents, industrial and metal dusts, and pigments and oils, to which these patients could have been exposed, could be the triggers for developing IgG4-SC.

\section{Clinical characteristics}

In our 527 patients, the most frequent symptom at presentation was jaundice, followed by pruritus and abdominal pain. Of note, it was extremely rare for patients with IgG4-SC to present with symptoms of decompensated cirrhosis, as variceal rupture was noted in only one patient, and there were no patients with ascites or encephalopathy. Since no treatment had been given to these patients, the natural course of IgG4-SC may be benign with very exceptional cases progressing to cirrhosis. Regarding laboratory data at presentation, the levels of serum alkaline phosphatase (ALP) were elevated in the majority of patients.

Table 2. Demographic Features of IgG4-SC

\begin{tabular}{ccccc}
\hline Region & Year & No. & Male sex, \% & $\begin{array}{c}\text { Age at pre- } \\
\text { sentation, yr }\end{array}$ \\
\hline USA $^{18}$ & 2008 & 53 & 85 & $62^{*}$ \\
UK $^{21}$ & 2014 & 68 & 74 & $61^{\dagger}$ \\
Japan $^{5}$ & 2017 & 527 & 83 & $66^{\dagger}$ \\
\hline
\end{tabular}

IgG4-SC, IgG4-related sclerosing cholangitis.

*Mean; ${ }^{\dagger}$ Median.

Table 1. Clinical Diagnostic Criteria of IgG4-Related Sclerosing Cholangitis, as Established by the Japanese Biliary Association in 2012

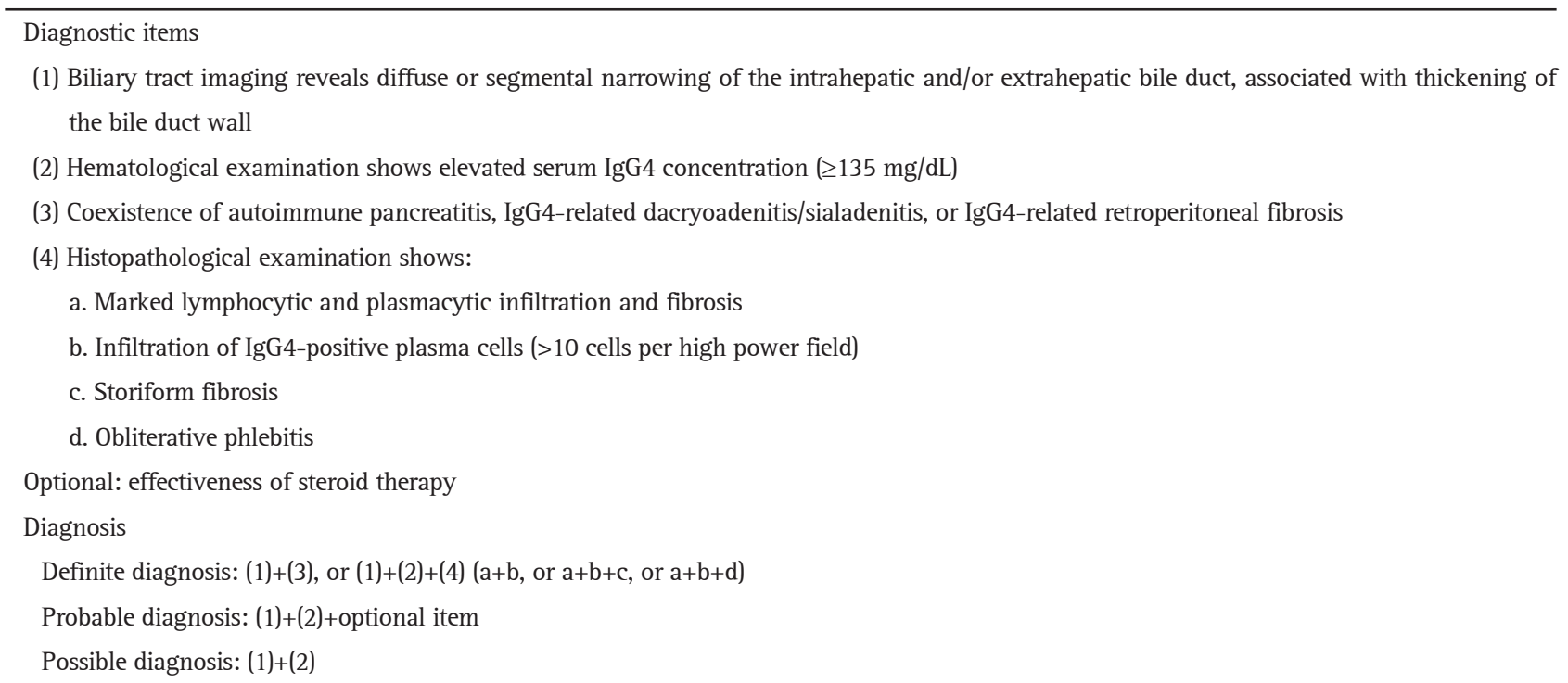

Adapted from Ohara H et al. J Hepatobiliary Pancreat Sci 2012;19:536-542, with permission from John Wiley and Son. ${ }^{15}$ 
Serum IgG4 levels were recorded for 485 of 535 patients with IgG4-SC and were above normal range $(<135 \mathrm{mg} / \mathrm{dL})$ in 409 patients (84.3\%); thus, serum IgG4 levels were within normal limits in $15.7 \%$ of patients with IgG4-SC (Fig. 2A). As discussed below, serum IgG4 levels were elevated in $12.5 \%$ of patients with PSC, and thus the serum IgG4 level was unable to uniquely differentiate PSC from IgG4-SC. Serum CA19-9 levels (normal range; $<37 \mathrm{IU} / \mathrm{mL}$ ) were elevated in 192 of 468 patients tested (41\%) and anti-nuclear antibodies were found in 137 of 354 patients tested (39\%).

Cholangiograms were available for 518 of 527 patients. According to the classification proposed by Nakazawa et al., ${ }^{23}$ intrapancreatic biliary strictures resembling pancreatic cancer (type 1) without any other strictures in the bile ducts represented the most common finding (64\%), followed by strictures in the hilar hepatic lesion (type 4, 10\%), and in both intrapancreatic and hilar lesions (type 3, 11\%). Intrahepatic segmental (type 2a) and diffuse (type $2 b$ ) strictures, in addition to intrapancreatic biliary strictures, were detected in 24 and 41 patients (5\% and 8\%), respectively. Unclassified cholangiographic findings were reported in 21 patients (4\%). Histological examination of the liver and bile duct were performed in 57 and 238 patients, respectively. No histological specimens were obtained from either the liver or the bile duct for a total of 179 patients.

\section{Comorbidities}

Presence of OOI of IgG4-RD greatly facilitates the diagnosis of IgG4-SC, and AIP was the most prevalent OOI being present in $88 \%$ of patients. Other 00Is included dacryoadenitis and sialadenitis in 73 patients (15\%), as well as involvement of the retroperitoneum (37 patients, 7\%), kidneys (six patients), aorta (six patients), and lungs (four patients).

Gallstones and gallbladder polyps were detected in 47 (10\%) and in 17 (3\%) of patients, respectively. Regarding malignant diseases in the biliary tract, the development of cholangiocarcinoma was only reported in four cases $(0.8 \%)$, indicating the oc-
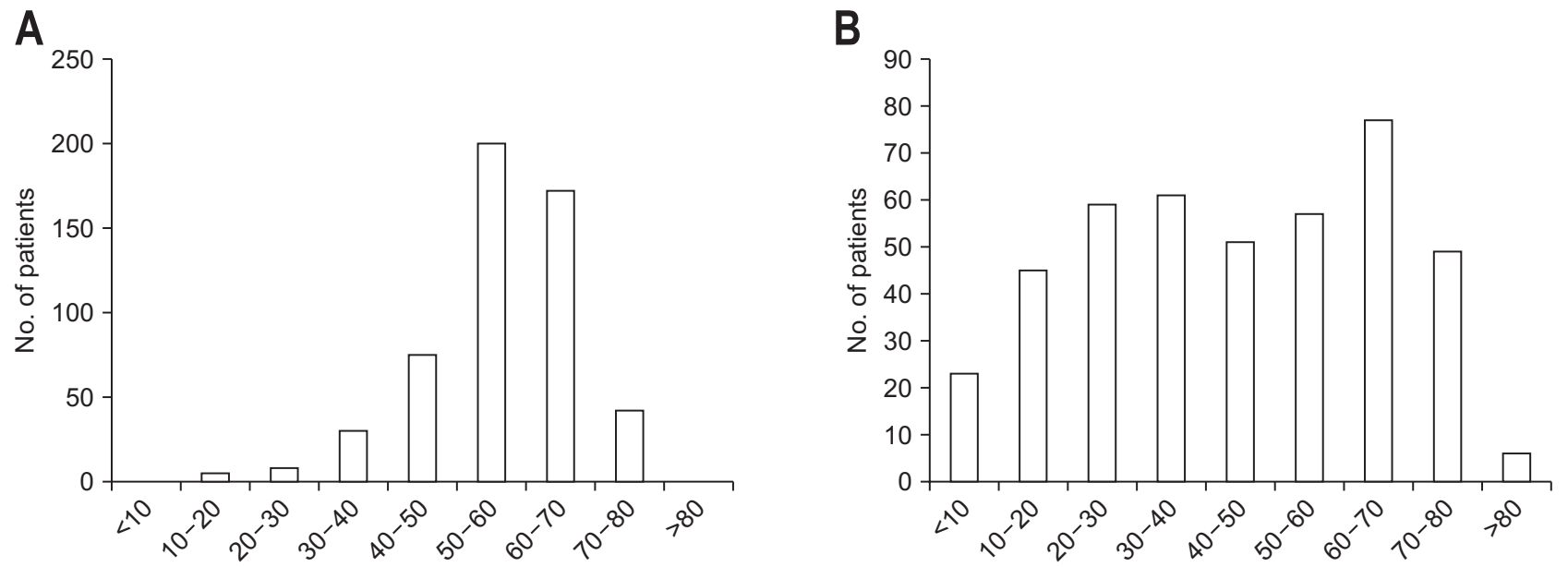

Fig. 1. Age distribution at presentation. (A) IgG4-related sclerosing cholangitis and (B) primary sclerosing cholangitis.
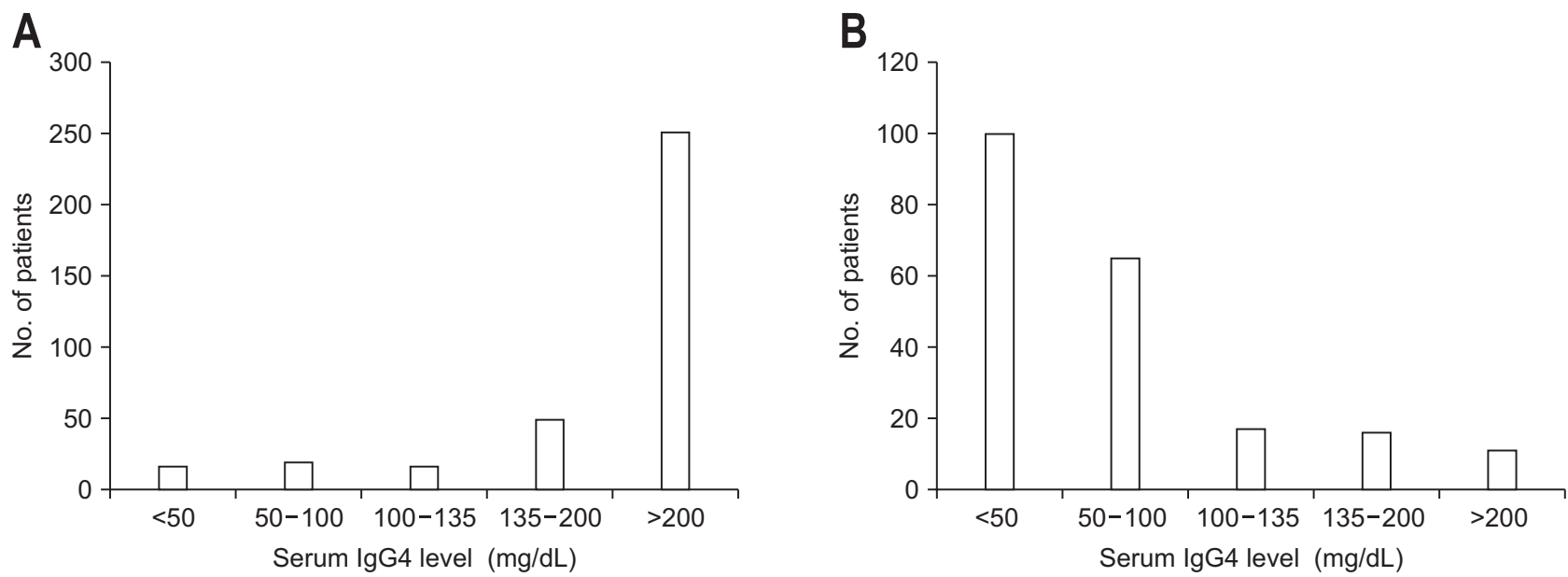

Fig. 2. Distribution of serum IgG4 levels at presentation. (A) IgG4-related sclerosing cholangitis and (B) primary sclerosing cholangitis. 
currence of cholangiocarcinoma is a rare event in patients with IgG4-SC.

\section{Management and outcomes}

It is well known that prednisolone (PSL) is a very effective treatment for IgG4-SC, as for other IgG4-RD. In our case series from Japan, PSL was initiated in 462 patients (88\%) following diagnosis. At the final follow-up, PSL treatment was terminated or continued in $312(63 \%)$ and 181 (37\%) patients, respectively. Overall, the treatment responses to these protocols were excellent. Reduction in ALP levels to $<50 \%$ of pretreatment levels or to within normal range was achieved in 395 patients $(88 \%$ of documented cases), and alleviation of biliary strictures was noted on the imaging results of 376 patients (90\% of documented cases). Endoscopic dilatation or stenting were performed in 48 (9\%) and 253 (50\%) patients, respectively. Surgical procedures were performed in 45 patients (9\%) and malignant disease was suspected preoperatively in most cases referred for surgery.

During $4.1 \pm 3.1$ years of follow-up, 27 patients (5\%) were reported to be deceased, but only four patients died due to liver or bile duct-related conditions. No liver transplantation (LT) was performed in this cohort. Cirrhosis progression accounted for two deaths. The overall 5- and 10-year survival rates were $94.4 \%$ and $81.0 \%$, respectively, and the 5- and 10-year survival-free from death due to liver or bile duct-related diseases were 98.9\% and 97.7\%, respectively. Therefore, poor outcomes including mortality due to liver or bile duct disease and LT can be avoided with treatment with corticosteroids in most cases of IgG4-SC with excellent outcomes.

The outcome of patients with IgG4-SC has also been investigated in retrospective cohorts in the United States ${ }^{18}$ and the United Kingdom. ${ }^{21}$ Sample size, follow-up period, corticosteroid treatment, and outcomes are summarized in Table 3. Progression to cirrhosis was noted in 5.2\% in the UK cohort and 7.5\% in the US cohort, respectively. Mortality due to liver or bile duct complications was found in only one case in the US cohort, and in two cases (liver failure and cholangiocarcinoma) and one case that underwent LT in the United Kingdom.

\section{PRIMARY SCLEROSING CHOLANGITIS}

PSC is a chronic cholestatic disease of unknown etiology, which is characterized by progressive stenosis and destruction of the bile ducts due to diffuse inflammation and fibrosis, and which eventually results in cirrhosis and liver failure. ${ }^{24,25} \mathrm{Un}$ fortunately, despite the severity of this disease, no standardized medical treatment is currently available, and although autoimmune reactions are believed to play a role in PSC pathogenesis, immunosuppressive therapy has not been effective. ${ }^{26-29}$ As a result, the long-term outcome for patients with PSC is unfavorable and is characterized by a high mortality rate, with LT being the only option for patients with advanced PSC.

\section{Epidemiology and demographics}

The prevalence and incidence of PSC are high in North Europe and North America, and low in Japan (Table 4). ${ }^{5,30-38}$ The prevalence is reported to be 4.15 to 16.2 per 100,000 population in Northern Europe and North America, ${ }^{39}$ respectively, while the prevalence of PSC was 0.95 per 100,000 in Japan. The prevalence of PSC is increasing somewhat in European countries, ${ }^{39}$ although it is unclear whether the apparent increase is due to a "real" increase of prevalence or to a better identification of the disease.

The nationwide surveys undertaken in 2015 in Japan enrolled 435 cases, which consisted of 263 males (60.5\%); the median age was 44.6 years old, ranging from 3 to 84 years (Fig. 1B). Male dominance is also consistently observed in Western countries (Table 4). It is of note that there were two peaks in the age distribution, 20 to 40 and 60 to 70 years (Fig. 1B), while individuals between 20 and 30 years old have been reported to be at high risk in Western countries. Nevertheless, two peaks in the age distribution were recently found in Canada and the United States (California). ${ }^{34,37}$

\section{Clinical characteristics}

In Japan, 62\% of patients were diagnosed as having PSC with no evidence of any symptoms, but with an elevation in liver enzymes detected on chance blood examinations. Among patients with symptoms, the most prevalent symptom was jaundice, fol-

Table 3. Treatment and Outcomes of Patients with IgG4-SC

\begin{tabular}{|c|c|c|c|c|c|c|c|c|}
\hline Region & Year & $\begin{array}{l}\text { Number of en- } \\
\text { rolled patients }\end{array}$ & $\begin{array}{l}\text { Follow-up } \\
\text { period, mo }\end{array}$ & $\begin{array}{l}\text { Corticosteroid } \\
\text { treatment }\end{array}$ & Cirrhosis & $\begin{array}{l}\text { All-cause } \\
\text { mortality }\end{array}$ & LT & $\begin{array}{l}\text { Mortality due } \\
\text { to liver and bile } \\
\text { duct diseases }\end{array}$ \\
\hline $\mathrm{USA}^{18}$ & 2008 & 53 & $29.5^{*}$ & $30(57)$ & $4(7.5)$ & 7 (13) & 0 & $1(1.9)$ \\
\hline $\mathrm{UK}^{21}$ & 2014 & 68 & 32.5 & $98(85)^{\dagger}$ & $6(5.2)^{*}$ & $11(9.6)^{*}$ & 1 & $3(2.6)^{*}$ \\
\hline Japan $^{5}$ & 2017 & 527 & 49.2 & $458(88)$ & NA & $26(5)$ & 0 & $4(0.8)$ \\
\hline
\end{tabular}

Data are presented as mean or number (\%).

IgG4-SC, IgG4-related sclerosing cholangitis; LT, liver transplantation; NA, not applicable.

*The mean follow-up period of patients treated with corticosteroids; ${ }^{\dagger}$ The proportion of 115 patients with autoimmune pancreatitis, including those without IgG4-SC. 
lowed by pruritus. While both serum ALP and gamma-glutamyl transpeptidase (GGT) levels were elevated in typical cases, ALP levels were less than 2x upper normal limits in 45\% of patients, indicating the possibility that PSC cannot be ruled out even in patients with relatively lower ALP levels. As described, serum IgG4 level was elevated in 12.5\% of patients with PSC (Fig. 2B). Histopathologically, an obliterative, non-suppurative cholangitis with substantial periductular fibrosis, referred as "onion-skin fibrosis," is a typical finding, although this finding is found only in $20 \%$ to $40 \%$ of patients.

The most useful diagnostic tool for PSC is cholangiography. Typical findings of PSC include a beaded appearance (Fig. 3A), pruned tree appearance (Fig. 3B), and band-like stricture (Fig. 3C). In addition, a shaggy appearance and diverticulum-like outpouching can be observed. While endoscopic retrograde cholangiography (ERC) is chosen as a diagnostic procedure in most cases in Japan, clinical guidelines of PSC from the European Society of Gastrointestinal Endoscopy and the European
Association for the Study of the Liver (EASL) recommend magnetic resonance cholangiography to avoid complications due to ERC. ${ }^{40}$

\section{Comorbidities}

Inflammatory bowel diseases (IBD) are frequently present as a comorbidity in patients with PSC. In previous reports, 50\% to $80 \%$ of patients with PSC in Western countries and 40\% of all patients (and 61\% in younger patients) in Japan had coexistent IBD. Ulcerative colitis is more common than Crohn's disease and atypical ulcerative colitis with right-sided colitis and rectum sparing is a characteristic feature of IBD in patients with PSC. The risk of colorectal carcinoma is higher in patients with IBD compared to those without IBD. ${ }^{41}$ Colonoscopy is mandatory in all patients diagnosed as PSC, despite the presence or absence of gastrointestinal symptoms, such as diarrhea or bloody stool.

PSC is associated with an increased risk of cholangiocarcinoma and gallbladder carcinoma, and is 400 -fold higher than

Table 4. Epidemiology of Primary Sclerosing Cholangitis

\begin{tabular}{lcccccc}
\hline \multicolumn{1}{c}{ Region } & No. & Incidence* & Prevalence* & Male sex, \% & $\begin{array}{c}\text { Presence of } \\
\text { IBD, \% }\end{array}$ & Year \\
\hline Norway $^{30}$ & 17 & 1.3 & 8.5 & 71 & 71 & 1998 \\
Singapore $^{31}$ & 10 & - & 1.3 & 90 & 20 & 2002 \\
USA (Minnesota) & 22 & 0.9 & 13.6 & 68 & 73 & 2003 \\
UK $^{33}$ & 46 & 0.91 & 12.7 & 62 & 62 & 2004 \\
Canada $^{34}$ & 49 & 0.92 & - & 55 & 67 & 2007 \\
UK $^{35}$ & 223 & 0.41 & 3.85 & 63.5 & 48 & 2008 \\
Sweden $^{36}$ & 199 & 1.22 & 16.2 & 71 & 76 & 2010 \\
USA (California) $^{37}$ & 169 & - & 4.15 & 59.8 & 64.5 & 2011 \\
UK $^{38}$ & 837 & 0.68 & 5.58 & 63.2 & 54.0 & 2017 \\
Japan $^{5}$ & 415 & - & 0.95 & 60.5 & 40 & $2008 / 2015$ \\
\hline
\end{tabular}

IBD, inflammatory bowel diseases.

*Per 100,000 population.
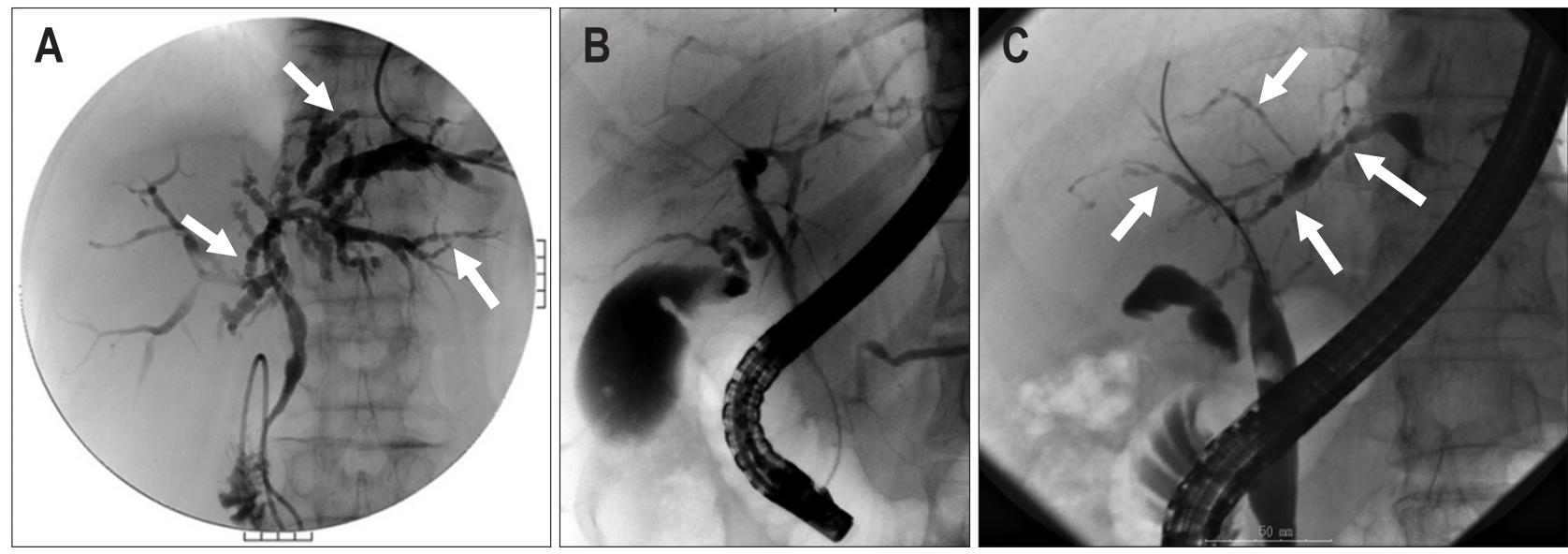

Fig. 3. Typical cholangiographic features of primary sclerosing cholangitis. (A) Beaded appearance (arrows), (B) pruned tree appearance, and (C) band-like stricture (arrows). 
healthy individuals. In Japan, 27 and four cases with PSC developed cholangiocarcinoma and gallbladder carcinoma, respectively, during follow-up, with an annual incidence of $1.43 \%{ }^{4}$ Cholangiocarcinoma can occur as an intrahepatic mass or a hilar tumor. Since one-third or half of cholangiocarcinomas are diagnosed at the same time, or within the first year after, diagnosis of PSC, it is therefore strongly advisable to carefully exclude the possibility of cholangiocarcinoma when a new diagnosis of PSC is made. Nevertheless, it could be extremely difficult to differentiate cholangiocarcinoma from PSC. Although novel modalities have been developed, including fluorescence in situ hybridization or probe-based confocal laser endomicroscopy, meta-analysis has demonstrated that single-operator cholangioscopy with targeted biopsies appears to be the most accurate endoscopic retrograde cholangiopancreatography (ERCP)based modality for diagnosing cholangiocarcinoma in PSC. ${ }^{42}$

\section{Management and outcomes}

To date, no medical treatment has been approved that improves transplant-free outcomes of patients with PSC in an evidence-based manner. Although ursodeoxycholic acid (UDCA) is currently used for a number of patients with PSC, clinical trials of UDCA failed to demonstrate a positive result from UDCA treatment and even a worse prognosis with a high-dose (>28 $\mathrm{mg} / \mathrm{kg} /$ day) UDCA. ${ }^{43}$ Thus, the clinical practice guidelines (CPG) for PSC from the American Association for the Study of the Liver did not recommend the use of UDCA in adult patients with PSC as medical therapy. ${ }^{44}$ The use of UDCA was not recommended in other CPGs for PSC from the EASL and from the American College of Gastroenterology. ${ }^{44,45}$ Meanwhile, several cohort studies, although retrospective, indicated that a reduction of ALP levels at 6 months or 1 year after commencement of UDCA treatment is associated with an improvement of longterm outcome of patients with PSC. ${ }^{46-49}$ The presence of dominant strictures, defined as a stenosis of the common duct with a diameter of $1.5 \mathrm{~mm}$ or less and/or $1.0 \mathrm{~mm}$ or less of a hepatic duct (within $2 \mathrm{~cm}$ of the bifurcation), requires endoscopic interventions which significantly improve long-term outcomes. ${ }^{50,51}$ LT is the only curative procedure for PSC but recurrence is not uncommon, especially with a living-donor. ${ }^{52}$

In a nationwide survey in Japan, the 5- and 10-year overall survival rates were $81.3 \%$ and $69.9 \%$, respectively, and the 5- and 10-year LT-free survival rates were 77.4\% and 54.9\%, respectively. ${ }^{4}$ Prognostic factors associated with worse outcomes included older age, lower albumin levels, and higher bilirubin levels.

\section{CONCLUSIONS}

Although the clinical presentation of both IgG4-SC and PSC includes similar cholestatic features, comorbidities, treatment response, and outcomes differ. Patients with IgG4-SC respond well to PSL treatment and long-term outcomes are excellent with very low rates for occurrence of cholangiocarcinoma. In contrast, there has been no standardized medical treatment approved for PSC to improve long-term outcomes and indeed almost half of patients with PSC faced death or LT in a Japanese cohort. In addition, the impact of comorbidities including IBD and cholangiocarcinoma is significant in PSC. Therefore, when patients with cholestatic features present in daily practices, it is strongly advisable to make an accurate diagnosis employing all available diagnostic modalities, and also to differentiate cholangiocarcinoma from IgG4-SC and PSC. The development of novel diagnostic procedures for IgG4-SC, PSC, and cholangiocarcinoma, as well as the development of new drugs for PSC are definitely unmet and urgent needs in this field.

\section{CONFLICTS OF INTEREST}

No potential conflict of interest relevant to this article was reported.

\section{REFERENCES}

1. Lazaridis KN, LaRusso NF. Primary sclerosing cholangitis. N Engl J Med 2016;375:1161-1170.

2. Karlsen TH, Folseraas T, Thorburn D, Vesterhus M. Primary sclerosing cholangitis: a comprehensive review. J Hepatol 2017;67:1298-1323.

3. Nakazawa T, Naitoh I, Hayashi K, Miyabe K, Simizu S, Joh T. Diagnosis of IgG4-related sclerosing cholangitis. World J Gastroenterol 2013;19:7661-7670.

4. Tanaka A, Tazuma S, Nakazawa T, et al. No negative impact of serum IgG4 levels on clinical outcome in 435 patients with primary sclerosing cholangitis from Japan. J Hepatobiliary Pancreat Sci 2017;24:217-225.

5. Tanaka A, Tazuma S, Okazaki K, et al. Clinical features, response to treatment, and outcomes of IgG4-related sclerosing cholangitis. Clin Gastroenterol Hepatol 2017;15:920-926.e3.

6. Hubers LM, Maillette de Buy Wenniger LJ, Doorenspleet ME, et al. IgG4-associated cholangitis: a comprehensive review. Clin Rev Allergy Immunol 2015;48:198-206.

7. Deshpande V, Zen Y, Chan JK, et al. Consensus statement on the pathology of IgG4-related disease. Mod Pathol 2012;25:11811192

8. Khosroshahi A, Wallace ZS, Crowe JL, et al. International consensus guidance statement on the management and treatment of IgG4-related disease. Arthritis Rheumatol 2015;67:1688-1699.

9. Stone JH, Khosroshahi A, Deshpande V, et al. Recommendations for the nomenclature of IgG4-related disease and its individual organ system manifestations. Arthritis Rheum 2012;64:3061-3067.

10. Umehara H, Okazaki K, Masaki Y, et al. A novel clinical entity, IgG4-related disease (IgG4RD): general concept and details. Mod Rheumatol 2012;22:1-14. 
11. Umehara H, Okazaki K, Masaki Y, et al. Comprehensive diagnostic criteria for IgG4-related disease (IgG4-RD), 2011. Mod Rheumatol 2012;22:21-30.

12. Okazaki K, Uchida K, Ikeura T, Takaoka M. Current concept and diagnosis of IgG4-related disease in the hepato-bilio-pancreatic system. J Gastroenterol 2013;48:303-314.

13. Hamano H, Kawa S, Horiuchi A, et al. High serum IgG4 concentrations in patients with sclerosing pancreatitis. N Engl J Med 2001;344:732-738.

14. Kamisawa T, Funata N, Hayashi Y, et al. A new clinicopathological entity of IgG4-related autoimmune disease. J Gastroenterol 2003;38:982-984.

15. Ohara H, Okazaki K, Tsubouchi H, et al. Clinical diagnostic criteria of IgG4-related sclerosing cholangitis 2012. J Hepatobiliary Pancreat Sci 2012;19:536-542.

16. Beuers U, Hubers LM, Doorenspleet M, et al. IgG4-associated cholangitis: a mimic of PSC. Dig Dis 2015;33 Suppl 2:176-180.

17. Doorenspleet ME, Hubers LM, Culver EL, et al. Immunoglobulin G4(+) B-cell receptor clones distinguish immunoglobulin G 4-related disease from primary sclerosing cholangitis and biliary/pancreatic malignancies. Hepatology 2016;64:501-507.

18. Ghazale A, Chari ST, Zhang L, et al. Immunoglobulin G4-associated cholangitis: clinical profile and response to therapy. Gastroenterology 2008;134:706-715.

19. Chari ST, Smyrk TC, Levy MJ, et al. Diagnosis of autoimmune pancreatitis: the Mayo Clinic experience. Clin Gastroenterol Hepatol 2006;4:1010-1016.

20. Kanno A, Masamune A, Okazaki K, et al. Nationwide epidemiological survey of autoimmune pancreatitis in Japan in 2011. Pancreas 2015;44:535-539.

21. Huggett MT, Culver EL, Kumar M, et al. Type 1 autoimmune pancreatitis and IgG4-related sclerosing cholangitis is associated with extrapancreatic organ failure, malignancy, and mortality in a prospective UK cohort. Am J Gastroenterol 2014;109:1675-1683.

22. de Buy Wenniger LJ, Culver EL, Beuers U. Exposure to occupational antigens might predispose to IgG4-related disease. Hepatology 2014;60:1453-1454.

23. Nakazawa T, Naitoh I, Hayashi K, et al. Diagnostic criteria for IgG4-related sclerosing cholangitis based on cholangiographic classification. J Gastroenterol 2012;47:79-87.

24. Karlsen TH, Schrumpf E, Boberg KM. Primary sclerosing cholangitis. Best Pract Res Clin Gastroenterol 2010;24:655-666.

25. Hirschfield GM, Karlsen TH, Lindor KD, Adams DH. Primary sclerosing cholangitis. Lancet 2013;382:1587-1599.

26. Giljaca V, Poropat G, Stimac D, Gluud C. Glucocorticosteroids for primary sclerosing cholangitis. Cochrane Database Syst Rev 2010;(1):CD004036.

27. Parkes M, Booth JC, Pillai G, Mee AS. Do steroids help jaundice caused by primary sclerosing cholangitis? J Clin Gastroenterol 2001;33:319-322.

28. Weismüller TJ, Wedemeyer J, Kubicka S, Strassburg CP, Manns MP. The challenges in primary sclerosing cholangitis--aetiopatho- genesis, autoimmunity, management and malignancy. J Hepatol 2008;48 Suppl 1:S38-S57.

29. Worthington J, Cullen S, Chapman R. Immunopathogenesis of primary sclerosing cholangitis. Clin Rev Allergy Immunol 2005;28:93-103.

30. Boberg KM, Aadland E, Jahnsen J, Raknerud N, Stiris M, Bell H. Incidence and prevalence of primary biliary cirrhosis, primary sclerosing cholangitis, and autoimmune hepatitis in a Norwegian population. Scand J Gastroenterol 1998;33:99-103.

31. Ang TL, Fock KM, Ng TM, Teo EK, Chua TS, Tan JY. Clinical profile of primary sclerosing cholangitis in Singapore. J Gastroenterol Hepatol 2002;17:908-913.

32. Bambha K, Kim WR, Talwalkar J, et al. Incidence, clinical spectrum, and outcomes of primary sclerosing cholangitis in a United States community. Gastroenterology 2003;125:1364-1369.

33. Kingham JG, Kochar N, Gravenor MB. Incidence, clinical patterns, and outcomes of primary sclerosing cholangitis in South Wales, United Kingdom. Gastroenterology 2004;126:1929-1930.

34. Kaplan GG, Laupland KB, Butzner D, Urbanski SJ, Lee SS. The burden of large and small duct primary sclerosing cholangitis in adults and children: a population-based analysis. Am J Gastroenterol 2007;102:1042-1049.

35. Card TR, Solaymani-Dodaran M, West J. Incidence and mortality of primary sclerosing cholangitis in the UK: a population-based cohort study. J Hepatol 2008;48:939-944.

36. Lindkvist B, Benito de Valle M, Gullberg B, Björnsson E. Incidence and prevalence of primary sclerosing cholangitis in a defined adult population in Sweden. Hepatology 2010;52:571-577.

37. Toy E, Balasubramanian S, Selmi C, Li CS, Bowlus CL. The prevalence, incidence and natural history of primary sclerosing cholangitis in an ethnically diverse population. BMC Gastroenterol 2011;11:83.

38. Liang H, Manne S, Shick J, Lissoos T, Dolin P. Incidence, prevalence, and natural history of primary sclerosing cholangitis in the United Kingdom. Medicine (Baltimore) 2017;96:e7116.

39. Boonstra K, Beuers U, Ponsioen CY. Epidemiology of primary sclerosing cholangitis and primary biliary cirrhosis: a systematic review. J Hepatol 2012;56:1181-1188.

40. Aabakken L, Karlsen TH, Albert J, et al. Role of endoscopy in primary sclerosing cholangitis: European Society of Gastrointestinal Endoscopy (ESGE) and European Association for the Study of the Liver (EASL) clinical guideline. Endoscopy 2017;49:588-608.

41. Palmela C, Peerani F, Castaneda D, Torres J, Itzkowitz SH. Inflammatory bowel disease and primary sclerosing cholangitis: a review of the phenotype and associated specific features. Gut Liver 2018;12:17-29.

42. Njei B, McCarty TR, Varadarajulu S, Navaneethan U. Systematic review with meta-analysis: endoscopic retrograde cholangiopancreatography-based modalities for the diagnosis of cholangiocarcinoma in primary sclerosing cholangitis. Aliment Pharmacol Ther 2016;44:1139-1151.

43. Lindor KD, Kowdley KV, Luketic VA, et al. High-dose ursodeoxy- 
cholic acid for the treatment of primary sclerosing cholangitis. Hepatology 2009;50:808-814.

44. Chapman R, Fevery J, Kalloo A, et al. Diagnosis and management of primary sclerosing cholangitis. Hepatology 2010;51:660-678.

45. Lindor KD, Kowdley KV, Harrison ME; American College of Gastroenterology. ACG clinical guideline: primary sclerosing cholangitis. Am J Gastroenterol 2015;110:646-659.

46. Lindström L, Hultcrantz R, Boberg KM, Friis-Liby I, Bergquist A. Association between reduced levels of alkaline phosphatase and survival times of patients with primary sclerosing cholangitis. Clin Gastroenterol Hepatol 2013;11:841-846.

47. Al Mamari S, Djordjevic J, Halliday JS, Chapman RW. Improvement of serum alkaline phosphatase to $<1.5$ upper limit of normal predicts better outcome and reduced risk of cholangiocarcinoma in primary sclerosing cholangitis. J Hepatol 2013;58:329-334.

48. Rupp C, Rössler A, Halibasic E, et al. Reduction in alkaline phosphatase is associated with longer survival in primary sclerosing cholangitis, independent of dominant stenosis. Aliment Pharmacol Ther 2014;40:1292-1301.

49. Stanich PP, Björnsson E, Gossard AA, Enders F, Jorgensen R, Lindor KD. Alkaline phosphatase normalization is associated with better prognosis in primary sclerosing cholangitis. Dig Liver Dis 2011;43:309-313.

50. Gluck M, Cantone NR, Brandabur JJ, Patterson DJ, Bredfeldt JE, Kozarek RA. A twenty-year experience with endoscopic therapy for symptomatic primary sclerosing cholangitis. J Clin Gastroenterol 2008;42:1032-1039.

51. Gotthardt DN, Rudolph G, Klöters-Plachky P, Kulaksiz H, Stiehl A. Endoscopic dilation of dominant stenoses in primary sclerosing cholangitis: outcome after long-term treatment. Gastrointest Endosc 2010;71:527-534.

52. Egawa H, Ueda Y, Ichida T, et al. Risk factors for recurrence of primary sclerosing cholangitis after living donor liver transplantation in Japanese registry. Am J Transplant 2011;11:518-527. 